\title{
Maternal interpersonal affiliation is associated with adolescents' brain structure and reward processing
}

\author{
S Schneider ${ }^{1}$, S Brassen ${ }^{1}$, U Bromberg ${ }^{1}$, T Banaschewski ${ }^{2}$, P Conrod ${ }^{3,4}$, H Flor ${ }^{2}$, J Gallinat ${ }^{5}$, Hugh Garavan ${ }^{6,7}$, \\ A Heinz ${ }^{5}$, J-L Martinot ${ }^{8,9}$, F Nees $^{2}$, M Rietschel ${ }^{2}$, MN Smolka ${ }^{10,11}$, A Ströhle $^{5}$, M Struve ${ }^{2}$, G Schumann ${ }^{3,12}$, C Büchel ${ }^{1}$ and \\ the IMAGEN consortium
}

Considerable animal and human research has been dedicated to the effects of parenting on structural brain development, focusing on hippocampal and prefrontal areas. Conversely, although functional imaging studies suggest that the neural reward circuitry is involved in parental affection, little is known about mothers' interpersonal qualities in relation to their children's brain structure and function. Moreover, gender differences concerning the effect of maternal qualities have rarely been investigated systematically. In 63 adolescents, we assessed structural and functional magnetic resonance imaging as well as interpersonal affiliation in their mothers. This allowed us to associate maternal affiliation with gray matter density and neural responses during different phases of the well-established Monetary Incentive Delay task. Maternal affiliation was positively associated with hippocampal and orbitofrontal gray matter density. Moreover, in the feedback of reward hit as compared with reward miss, an association with caudate activation was found. Although no significant gender effects were observed in these associations, during reward feedback as compared with baseline, maternal affiliation was significantly associated with ventral striatal and caudate activation only in females. Our findings demonstrate that maternal interpersonal affiliation is related to alterations in both the brain structure and reward-related activation in healthy adolescents. Importantly, the pattern is in line with typical findings in depression and post-traumatic stress disorder, suggesting that a lack of maternal affiliation might have a role in the genesis of mental disorders.

Translational Psychiatry (2012) 2, e182; doi:10.1038/tp.2012.113; published online 13 November 2012

\section{Introduction}

Much animal and human research has been conducted in an attempt to investigate effects of parenting on neural development and mental health. In a different field, interpersonal research, valuable concepts have been developed to describe human interaction and its relationship to attachment and psychopathology. However, a connection between these research areas has rarely been endeavored.

Maternal care affects stress reactivity and neural systems regulating stress responses. ${ }^{1,2}$ Chronically stressful environments adversely affect hippocampal plasticity, ${ }^{3-5}$ and particularly mother-offspring interaction is important for hippocampal structural development. ${ }^{6}$ Recently, a study in humans has established that early childhood maternal support predicts hippocampal volume as measured at school age. ${ }^{7}$ Moreover, hippocampal structure seems to be affected by attachment style. ${ }^{8}$ Functional imaging indicates that early-life stress is associated with dysfunctional neural reward processing. ${ }^{9}$ With parental affection being a substantial source of a child's reward, it is conceivable that the neural reward system, including striatum and orbitofrontal cortex (OFC), has an important role in positive mother-infant interaction and attachment. ${ }^{10-12}$ This is in line with the finding that a favorable disposition towards social relationships and attachment is associated with increased gray matter density in orbitofrontal and striatal regions in men. ${ }^{13}$ Corresponding structural changes, including the hippocampus and the prefrontal cortex, have been found in patients with major depressive disorder (MDD), ${ }^{14,15}$ which has been related to low parental affection. ${ }^{16,17}$ Furthermore, patients suffering from MDD have attenuated ventral striatal activation during reward anticipation ${ }^{18,19}$ as well as decreased ventral striatal and caudate activation during reward feedback. ${ }^{18}$ Even more compelling is that this pattern has also been observed in healthy adolescents at risk for depression. ${ }^{20,21}$ In samples free of psychiatric disorders, depressive symptoms and their

\footnotetext{
${ }^{1}$ Department of Systems Neuroscience, University Medical Center Hamburg-Eppendorf, Hamburg, Germany; ${ }^{2}$ Central Institute of Mental Health, Mannheim, Germany; ${ }^{3}$ Institute of Psychiatry, King's College London, London, United Kingdom; ${ }^{4}$ Department of Psychiatry, Université de Montréal, CHU Ste Justine Hospital, Montreal, QC, Canada; ${ }^{5}$ Department of Psychiatry and Psychotherapy, Campus Charité Mitte, Charité - Universitätsmedizin Berlin, Berlin, Germany; ${ }^{6}$ Institute of Neuroscience, Trinity College Dublin, Dublin, Ireland; ${ }^{7}$ Departments of Psychiatry and Psychology, University of Vermont, Burlington, VT, USA; ${ }^{8}$ Institut National de la Santé et de la Recherche Médicale, INSERM Unit 1000 'Imaging \& Psychiatry', SHFJ CEA, University Paris Sud, Orsay, Maison de Solenn, Paris, France; ${ }^{9}$ Department of Psychopathology and Adolescent Medicine, Paris Descartes University, Paris, France; ${ }^{10}$ Department of Psychiatry and Psychotherapy, Technische Universität Dresden, Dresden, Germany; ${ }^{11}$ Neuroimaging Center, Department of Psychology, Technische Universität Dresden, Dresden, Germany and ${ }^{12}$ MRC Social, Genetic and Developmental Psychiatry (SGDP) Centre, London, United Kingdom

Correspondence: S Schneider, Department of Systems Neuroscience, University Medical Center Hamburg-Eppendorf, Martinistr. 52, Hamburg 20246, Germany. E-mail: s.schneider@uke.uni-hamburg.de
}

Keywords: fMRI; gray matter; hippocampus; prefrontal cortex; striatum

Received 23 July 2012; revised 21 September 2012; accepted 6 October 2012 
increase are related to the neural response to reward. ${ }^{22,23}$ A similar picture emerges for post-traumatic stress disorder (PTSD), ${ }^{24,25}$ which can arise from traumatic experiences associated with adverse parenting.

Naturally, the interaction between a mother and her child is coined by the mother's general interpersonal style. Research in interpersonal theory ${ }^{26}$ has proposed that interpersonal behavior can be described by two dimensions, namely affiliation and dominance. ${ }^{27}$ In this concept, affiliation continuously ranges from cold-hostile to warm-loving, and dominance is described by dominating vs submissive behavior. It has been shown that friendly loving interpersonal style as assessed by the Inventory of Interpersonal Problems $(\text { IIP })^{28}$ or other interpersonal measures such as the Structural Analysis of Social Behavior ${ }^{29}$ is connected to secure attachment, while a cold-hostile style is related to dismissing and anxious attachment. ${ }^{30-33}$ Nevertheless, there are critical differences between the concepts of affiliation and attachment: while attachment represents the quality of the relationship between a person and a specific important other, interpersonal affiliation relates to a person's general extent of love and affection toward others. ${ }^{33}$ Thus, secure attachment can be seen as a result of experiencing an attachment figure as loving-friendly, ${ }^{33}$ making mothers' affiliation a prerequisite for their children's attachment that is not distorted by the child's perspective. As core behavioral-motivational processes underlying the trait of affiliation, different phases of reward processing have been suggested. ${ }^{34}$ With mothers' own attachment and social characteristics having a fundamental role in parenting and children's attachment, ${ }^{35-37}$ interpersonal behavior of mothers may directly relate to their children's neural development.

However, studies in healthy volunteers investigating how interpersonal qualities assessed in mothers relate to their children's brain structure and function are lacking. Therefore, we hypothesized that in healthy adolescents, low maternal affiliation would be associated with reduced gray matter density and attenuated activation during reward processing in the hippocampus, OFC and striatum. As many of the studies cited above have focused on either males or females, or have detected gender differences, ${ }^{13,20,24,38}$ we additionally sought to investigate whether male and female adolescents' neural properties are differentially affected by maternal affiliation.

\section{Materials and methods}

Participants. Participants were from the IMAGEN study (for a detailed description of the sample, see Schumann et $\left.a l^{39}\right)$. Written informed consent to participate in the study was obtained from the parents as well as assent from the participants themselves, and procedures were approved by the local ethics committee. As the questionnaire used for the affiliation assessment was only given to the parents accompanying participants assessed in Hamburg, Germany, between January and July 2010, only this subset was analyzed for the present study $(n=79)$. Eight datasets had to be excluded after quality control. Of the remaining 71 datasets, seven questionnaires had been filled out by fathers, all others had been filled out by mothers. To keep the sample as homogenous as possible, we investigated only the data from those participants whose mothers had filled out the questionnaire $(n=64)$. In order to identify and exclude participants with psychiatric disorders, clinical symptomatology was individually assessed with the computerized Development and Well-Being Assessment ${ }^{40}$ and then rated by experienced psychiatrists. This resulted in the exclusion of another participant and a final sample of $n=63$ without clinically relevant symptomatology (rating: 'sure no clinical disorder present'). Of this final sample, 26 were female, the mean (s.d.) age was $14.24(0.25)$ years, and according to the Puberty Development Scale ${ }^{41}$ the mean (s.d.) pubertal status was at $67(11) \%$ of the maximum score. The participants' intelligence was estimated by the sum score of five subtests of the Wechsler Intelligence Scale for Children IV ${ }^{42}$ (that is, block design, matrix reasoning, vocabulary, digit span and similarities), with a mean (s.d.) sum score of 189 (19) points. As the composite measure of these particular subtests is not a validated IQ measure, we refrain from converting it to the IQ scale. However, compared with the large sample of the 14-year-old IMAGEN participants assessed in Europe ( $n=2025$, mean (s.d.) sum score $=186$ (23) points), the present sample was in the normal range $(T(2086)=0.084, P=0.398)$.

Maternal affiliation and personality assessment. The mothers' interpersonal affiliation score was calculated from their scores on the German Version of the IIP. ${ }^{43}$ The IIP is a standard measure to assess problems that a person might encounter in interpersonal contact. $^{28}$ Importantly, the 64 items answered on a five-point scale capture general patterns in interpersonal situations (for example, 'I keep other people at distance too much'), not behavior toward a specific person. The IIP total score of the mothers in this sample (mean (s.d.) $=1.08(0.49)$ ) is comparable with the healthy norm for this age group. ${ }^{43}$ Based on the interpersonal theory of psychiatry, ${ }^{26}$ the IIP consists of eight scales arranged in a circumplex: Vindictive (BC), Cold (DE), Socially avoidant (FG), Nonassertive (HI), Exploitable (JK), Overly nurturant (LM), Intrusive (NO) and Domineering (PA). ${ }^{44}$ In correspondence with the findings by Leary, ${ }^{27}$ the circumplex of the ipsated (mean-subtracted) scale values is spanned by two dimensions: love/affiliation, characterized by friendly/ loving vs cold/hostile behavior, and dominance described by dominating vs submissive behavior. ${ }^{45-47}$ This structure closely corresponds to the universal structure of interpersonal measures. ${ }^{43,48}$ Even though the IIP is often used in a clinical context, it has been shown that the circumplex structure fits just as well in healthy adult samples. ${ }^{43}$ The mothers' IIP scale values were ipsated and individually $z$-transformed, then the affiliation scores were computed according to Horowitz et al. ${ }^{43}$ and Gurtman: ${ }^{45,46}$ affiliation $=0.25 \times((\mathrm{zLM})+0.707 \times(\mathrm{zNO})+0.707 \times(\mathrm{zJK})-$ $0.707 \times(z F G)-0.707 \times(z B C)-(z D E))$. While the IIP total score is indicative of a person's total amount of problematic interpersonal behavior, the affiliation score is based on ipsated values and thus does not represent problem behavior but rather a general interpersonal style, especially in a healthy sample. ${ }^{43,48}$ Please note that the term 'maternal interpersonal affiliation' does not specifically refer to the affiliation of 


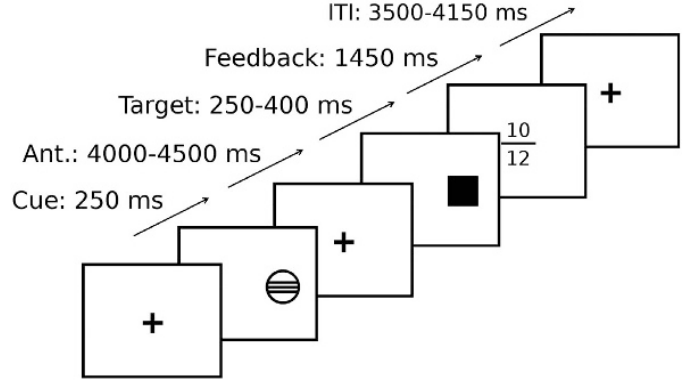

Figure 1 The time line of the Monetary Incentive Delay task ${ }^{49}$ used during functional imaging (here: a 'hit large reward' trial, cued by a circle with three lines, with the feedback screen indicating a win of 10 points in the present trial and 12 points total. For a detailed description of the task, see Methods). For the association with maternal interpersonal affiliation according to the Inventory of Interpersonal Problems, ${ }^{43}$ both anticipation and feedback vs baseline were investigated, as well as feedback of hit vs feedback of miss.

mothers toward their children but to the mothers' interpersonal style in general.

Reward task. In order to assess reward processing during functional magnetic resonance imaging (MRI), a modified version of the Monetary Incentive Delay task ${ }^{49}$ was used (Figure 1). Each trial consisted of anticipation, response and feedback. Before the anticipation phase, a cue signaled the position of the target as well as the type of reward that could be attained by a correct response. Different cues distinguished between large reward (10 points), small reward (two points) and no reward (zero points) conditions. After a random anticipation interval of 4 000-4 $500 \mathrm{~ms}$ length, the target appeared. Participants were instructed to respond to the target as quickly as possible via button press and informed that the points they earned would be converted into chocolate treats after scanning. The duration of the target appearance was continuously adapted to the performance of the subject, ensuring a successful performance on approximately $66 \%$ of all the trials. Immediately following the response, feedback indicated the number of points attained in the recent trial as well as the total points earned during the task. The inter-trial interval varied so that each trial took approximately 10,000 ms (Figure 1). Large, small, and no win conditions were randomized throughout the task (22 trials each, summing up to 66 trials in total).

Imaging parameters. All MRI data were acquired using a 3-Tesla Siemens Trio MRI scanner in Hamburg, Germany. High-resolution anatomical MRIs were acquired, including a three-dimensional T1-weighted magnetization-prepared gradient echo sequence based on the Alzheimer's Disease Neuroimaging Initiative protocol. The structural image comprised 160 slices $(1.1 \mathrm{~mm}$ thickness, TR $=2300$ $\mathrm{ms}, \mathrm{TE}=2.8 \mathrm{~ms}$ ) and required $9 \mathrm{~min}$ for acquisition. In the functional task, 300 volumes per subject were obtained, each comprising 40 slices. The slices were aligned to the connecting line between the anterior and posterior commissure $(2.4 \mathrm{~mm}$ thickness, $1 \mathrm{~mm}$ gap, $\mathrm{TR}=2200$ $\mathrm{ms}, \mathrm{TE}=30 \mathrm{~ms})$. The functional scanning procedure required $11 \mathrm{~min}$.
Functional and structural MRI preprocessing and analysis. All image analysis procedures were conducted using SPM8 (Wellcome Department of Cognitive Neurology, London, UK). For the voxel-based morphometry analysis, ${ }^{50}$ T1 images were segmented using the SPM8 segmentation toolbox, then modulated and normalized to Montreal Neurological Institute (MNI) space using the Diffeomorphic Anatomical Registration Through Exponentiated Lie Algebra (DARTEL) toolbox 6 as implemented in SPM8. The structural images were smoothed with a $7-\mathrm{mm}$ Gaussian isotropic kernel.

For the functional analysis, EPI (echo planar imaging) images were co-registered with the T1 structural image, then realigned and resliced. A first-level model was constructed on the unsmoothed single-subject data using each of the following regressors twice (for successful trials and for unsuccessful trials, respectively): (1) anticipation of large reward, (2) anticipation of small reward, (3) anticipation of no reward, (4) feedback large reward, (5) feedback small reward, and (6) feedback no reward. Trials in which subjects failed to respond were modeled separately. Individual movement parameters were included as covariates. Using DARTEL, we created a custom template from the participants' T1 images and normalized single-subject contrast images to $\mathrm{MNI}$ space, then smoothed with a $6-\mathrm{mm}$ Gaussian isotropic kernel.

Because of gender-specific effects of parental behavior on the children/offspring (see Introduction), we modeled maternal affiliation separately for each gender. In order to do so, a second-level model was conducted for both functional and structural data with maternal affiliation as covariate of interest modeled individually for each gender. This allowed us (1) to investigate potential gender interactions of maternal affiliation and (2) to model the association with maternal affiliation in all participants while controlling for potential gender differences. In all analyses, pubertal status ${ }^{41}$ and intelligence estimate ${ }^{42}$ were included as gender-specific nuisance covariates. There were several reasons to include pubertal status instead of age. Although all participants were within a very narrow age range (14 years), they largely differed in pubertal status. Moreover, at least in females, pubertal status predicts gray matter density in critical regions more accurately than age does, and sexual dimorphisms concerning gray matter density in these regions are driven more by pubertal influences than by age. ${ }^{51}$ In order to control for global volumetric differences between participants in the voxel-based morphometry analysis, total intracranial volume was also included as a nuisance covariate.

For all analyses, the significance threshold was set to $P<0.05$, using small volume family-wise error correction at the voxel level. Volumes of interest were the bilateral ventral striatum, caudate, hippocampus and frontal orbital cortex. Correction for the ventral striatum was based on an 18- $\mathrm{mm}$ diameter sphere centered at coordinates $\mathrm{x}= \pm 14, \mathrm{y}=8$, $z=-8$, as indicated in previous studies ${ }^{52-54}$ All other volumes of interest were taken from the Oxford Cortical/Subcortical Structural Atlas, with a probability threshold at 0.5. Figures are displayed at a threshold of $P<0.001$, uncorrected, for visualization purposes, and projected onto the participants' mean structural scan. 


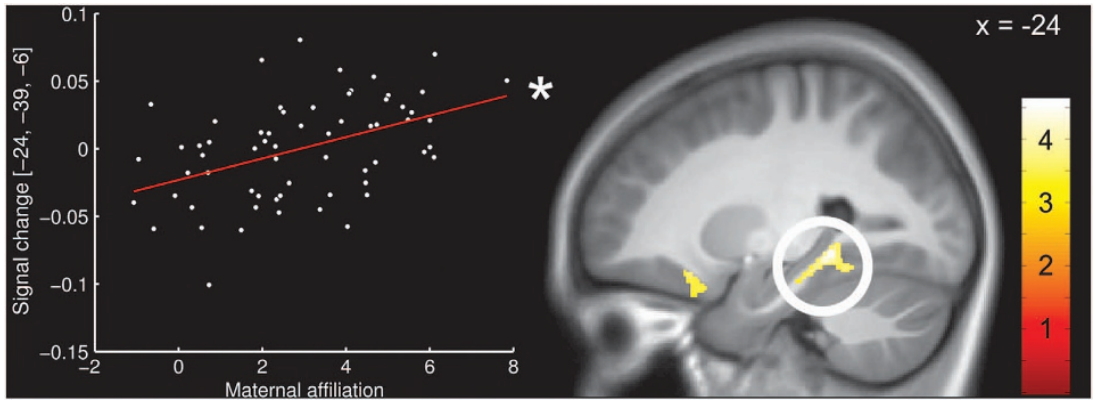

Figure 2 Associations between maternal interpersonal affiliation according to the Inventory of Interpersonal Problems ${ }^{43}$ and gray matter density in the hippocampus. The scatterplot represents individual maternal affiliation scores and intensity values at the peak voxel in the hippocampus. The color bar indicates $t$-values. The activations are displayed at a threshold of $P<0.001$, uncorrected, for visualization purposes. *Significant at $P<0.05$, corrected.

Table 1 Main effects of the Monetary Incentive Delay task ${ }^{49}$ on regional Blood Oxygenation Level Dependent (BOLD) response in the regions of interest (all $p<0.01$, corrected)

\begin{tabular}{|c|c|c|}
\hline Structure & $\begin{array}{l}\text { Voxeld } \\
T(57)\end{array}$ & $\begin{array}{c}\text { Coordinates } \\
x, y, z(m m)\end{array}$ \\
\hline \multicolumn{3}{|c|}{ Reward anticipation (large rewards) } \\
\hline Left ventral striatum & 11.52 & $-16,8,-3$ \\
\hline Right ventral striatum & 10.93 & $14,11,-6$ \\
\hline Left caudate & 7.60 & $-14,14,-2$ \\
\hline Right caudate & 7.95 & $12,12,-2$ \\
\hline Left hippocampus & 6.80 & $-21,-39,-3$ \\
\hline Right hippocampus & 6.07 & $27,-36,-6$ \\
\hline Left orbitofrontal cortex & 10.76 & $-32,27,-2$ \\
\hline Right orbitofrontal cortex & 11.05 & $32,21,-9$ \\
\hline \multicolumn{3}{|c|}{ Reward feedback (large rewards) } \\
\hline Left ventral striatum & 12.22 & $-10,6,-3$ \\
\hline Right ventral striatum & 10.68 & $12,11,-14$ \\
\hline Left caudate & 10.59 & $-10,9,-2$ \\
\hline Right caudate & 8.12 & $10,8,4$ \\
\hline Left hippocampus & 8.36 & $-22,-39,-5$ \\
\hline Right hippocampus & 8.36 & $30,-33,-11$ \\
\hline Left orbitofrontal cortex & 13.57 & $-33,26,-2$ \\
\hline Right orbitofrontal cortex & 12.51 & $38,21,-8$ \\
\hline \multicolumn{3}{|c|}{ Feedback hit > miss (large rewards) } \\
\hline Left ventral striatum & 6.79 & $-9,12,-11$ \\
\hline Right ventral striatum & 7.10 & $10,9,-6$ \\
\hline Left caudate & 4.99 & $-16,18,-5$ \\
\hline Right caudate & 4.87 & $10,14,-2$ \\
\hline Left hippocampus & 4.66 & $-22,-21,-20$ \\
\hline Right hippocampus & 4.16 & $22,-30,-8$ \\
\hline Left orbitofrontal cortex & 6.21 & $-16,12,-15$ \\
\hline Right orbitofrontal cortex & 5.17 & $14,12,-15$ \\
\hline
\end{tabular}

In order to attain the maximal sensitivity to detect associations with reward-related activation, we attempted to use the contrast with the most robust reward-related response for the association analyses. Previous findings show that reward magnitude is reflected in reward-related activation, with large rewards eliciting the greatest reward-related response. ${ }^{54}$ Thus, we focused the association analyses on the large reward condition, as it has been done previously. ${ }^{53}$

\section{Results}

Voxel-based morphometry analysis. In agreement with our hypotheses, we found higher maternal scores in the affiliation dimension to be associated with increased gray matter density in bilateral hippocampus (x, y, z: -24 ,
$-39,-6, T(53)=3.85, P=0.011$ and $20,-37,4, T(53)$ $=3.29, P=0.048$, both corrected; see Figure 2). Moreover, the affiliation dimension was associated with left orbitofrontal gray matter density $(-24,21,-18, T(53)=3.55, P=0.036$, corrected).

No significant gender interactions were observed in this analysis.

\section{Reward task}

Anticipation. As expected, the reward network was activated during the anticipation of large rewards, including the striatum, OFC and hippocampus (Table 1). For the whole-brain activation beyond the regions of interest, please refer to Supplementary Table S1.

Nevertheless, we neither found significant associations between maternal interpersonal affiliation and activation during reward anticipation in the hypothesized regions, nor did we find significant gender interactions.

Feedback. During feedback of large rewards, again all regions of interest were strongly activated (Table 1; for the whole-brain activation beyond the regions of interest, see Supplementary Table S1), and we found an association between maternal interpersonal affiliation and right ventral striatal activation $(15,3,0, T(55)=3.33, P=0.050$, corrected). However, testing for gender interactions in the effect of maternal affiliation revealed significant interactions in this area $(F(55)=18.70, P=0.008$, corrected) as well as in the right caudate $(18,21,-2, F(55)=19.61, P=0.006$, corrected). Post-hoc tests showed that in these regions, the association was stronger in females (Figure 3). When dividing the sample into a male and a female group and repeating the analysis in both groups separately, the association with maternal affiliation was significant in females (right striatum: $15,3,0, T(22)=3.81, P=0.042$ and right caudate: $18,21,-2, T(22)=4.76, P=0.008$, both corrected; Figure 3 ) but not in males.

Feedback hit vs miss. We then analyzed the differential activation between feedback of large reward hits (successful trials when participants' responses were fast enough and reward was delivered) and feedback of large reward misses (unsuccessful trials when responses were not fast enough and no reward was delivered). Also in this contrast, all regions of interest showed robust activation (Table 1 ). 


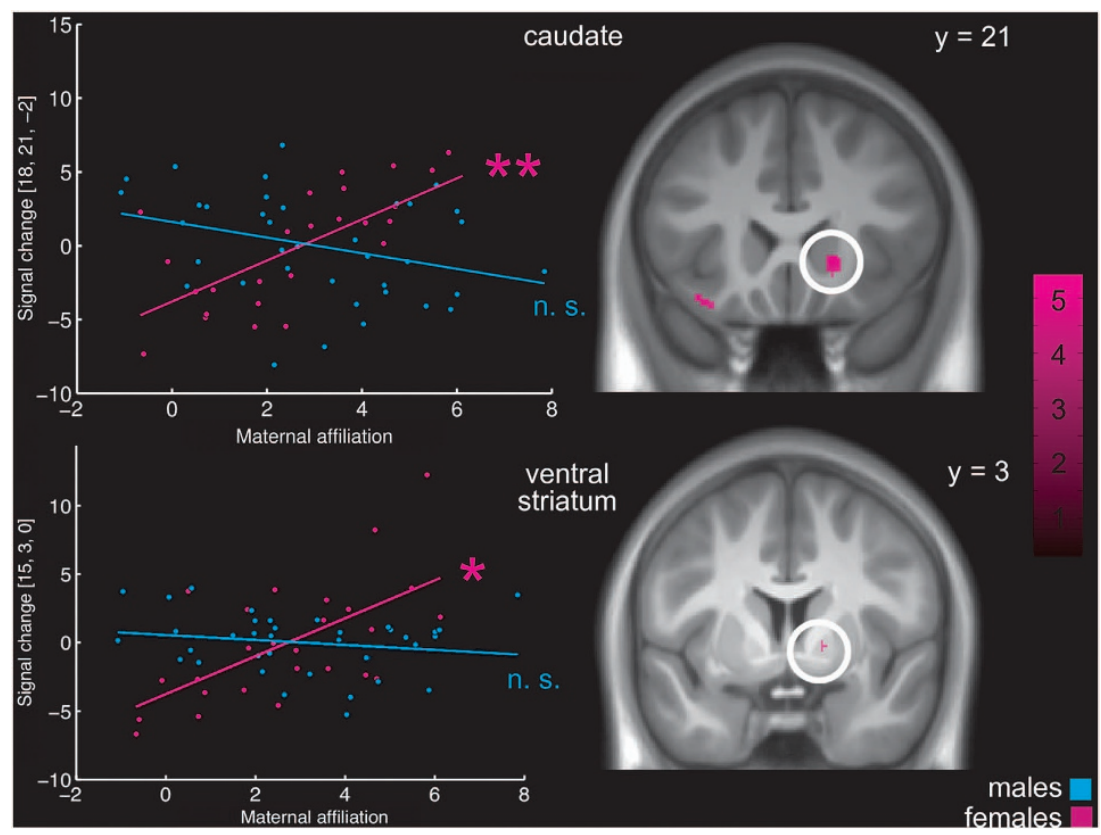

Figure 3 Gender-specific association between maternal interpersonal affiliation according to the Inventory of Interpersonal Problems ${ }^{43}$ and striatal activation in the Monetary Incentive Delay task ${ }^{49}$-feedback of large rewards. The scatterplot represents individual maternal affiliation scores and intensity values at the peak voxel in the caudate (upper part) and the ventral striatum (lower part). Blue dots represent values of males, pink dots represent values of females. The activation maps show the activation associated with maternal affiliation in the female subgroup. The color bar indicates $t$-values. The activations are displayed at a threshold of $P<0.001$, uncorrected, for visualization purposes. ${ }^{*}$ Significant at $P<.01$; ${ }^{*}$ significant at $P<0.05$, all corrected; NS, non-significant.

Maternal interpersonal affiliation was positively associated with the differential activation between large reward hits and misses in the left caudate $(-18,12,13, T(55)=3.41$, $P=0.043$, corrected). No significant gender interactions were observed.

\section{Discussion}

Our results demonstrate that interpersonal affiliation in mothers is associated with bilateral hippocampal and left orbitofrontal gray matter density in their adolescent children. Furthermore, during functional MRI with an established reward paradigm, partly gender-specific associations with feedback-related activation in the ventral and dorsal striatum were observed.

First of all, our results replicate and extend previous crosssectional $^{38,55}$ and longitudinal findings in humans ${ }^{7}$ and animals ${ }^{6}$ that relate maternal care or support to hippocampal gray matter volume. The convergence of outcomes attained with such different methodologies makes it likely that there is a predominant common basis underlying this link. Considering that hippocampal reductions have robustly been associated with environmental stress, ${ }^{3,5}$ it can be speculated that 'cold' or unsupportive mothers represent a stressful environment for children, thereby causing an impairment of their hippocampal development.

A more novel finding is the connection between maternal affiliation and gray matter density in the OFC, a region that has a central role in the concept of attachment. ${ }^{11}$ Moreover, theoretical accounts conceptualize affiliation in parents as a qualification for secure attachment in children, ${ }^{33}$ and neural processes involving the OFC have a role in both affiliation and attachment. $^{10,12,34}$ Thus, the OFC could be an important node, linking interpersonal affiliation in mothers and attachment in their children. As recent research suggests, reward learning occurs by building up representations of reward values through fine-grained activity patterns in response to specific cues in the OFC. ${ }^{56,57}$ It can be speculated that a 'cold', non-affiliative mother offering her child less emotional and social reward causes a suboptimal formation of neural value representations in the OFC. This might lead to a deficient structural development of the OFC, and consequently, motivational and hedonic value of emotional and social rewards would be monitored inefficiently. Possibly, such a process could be related to reduced motivation and ability to understand the intentions of others, in line with findings relating orbitofrontal volume to reward dependency and social competences. ${ }^{13,58,59}$ Furthermore, as our functional imaging findings are indicative of an impact on reward processing that goes beyond social reward and attachment, such deficiencies in reward processing may generalize to other types of reward.

Although it is unlikely that these volumetric differences reflect effects of neurogenesis, the basis of gray matter differences in healthy subjects measured by voxel-based morphometry cannot be clearly determined. ${ }^{60,61}$ Yet, animal studies give hints about potential biological mechanisms underlying our findings. In the rodent hippocampus, the consequences of early maternal separation or reduced maternal care include attenuated rates of synaptic development ${ }^{62}$ as well as disrupted cell proliferation and migration, likely influenced by an altered radial glia cell density and 
morphology. ${ }^{63}$ Also outside the hippocampus, specific processes as to how parental deprivation affects rodent brain structure have been identified, for example impaired dendritic and synaptic development of the apical dendrites in the OFC. ${ }^{64}$ Importantly, all these findings suggest that a lack of parental care relates to a structural deficit rather than to simple variability, but they do not converge on a single underlying process. A combination of different cellular mechanisms may be involved in experience-dependent gray matter variability, ${ }^{61}$ and this could also be the case for parenting-related effects.

In the functional imaging reward task, striatal regions were robustly activated during the main effects of reward anticipation and feedback, demonstrating that the task effectively elicited reward-related activation. Perhaps, the rewardrelated activation might have been an even more robust basis to detect individual differences had we used money instead of chocolate treats as rewards, which was not possible for ethical reasons. In response to large reward feedback, we found significant gender interactions in the association between ventral striatal and caudate activation and maternal interpersonal affiliation. Post-hoc analyses revealed that the association between activation and maternal affiliation was significant in the female but not in the male part of the sample. This observation is in line with previous reports that some effects of maternal care are only present in females. ${ }^{38,65}$ The region associated with maternal affiliation only in the female subgroup, the ventral striatum, has been suggested as key mediator of consummatory reward deficits, contributing to the experience of pleasure. ${ }^{66}$ In this sense, particularly daughters of rather 'cold' as compared with affiliative mothers might have deficits in the experience of pleasure. It could be that females are more vulnerable to maternal effects on striatal reward processing because of general gender differences in the functioning of reward-related areas. ${ }^{67,68}$ Another possibility is that the interaction with the same-sex parent has particularly strong effects on the development. This has been suggested by a questionnaire study finding a correlation between parenting style and interpersonal sensitivity only for the same-sex parent, particularly in females. ${ }^{69}$ Future studies are needed to clarify the exact nature of these gender differences. However, we found an association between maternal affiliation and the differential activation between feedback of large reward hits and misses in the left caudate that was not different in males and females. As suggested previously, reduced caudate activation in such a differential feedback contrast may relate to a deficit in reward learning in order to optimize goal-directed behavior. ${ }^{18,66}$

The pattern of structural and functional variation related to low maternal affiliation also resembles patterns observed in PTSD and MDD. ${ }^{14,18,25,70}$ Although our results may not directly support these studies as the present sample is free of clinical disorders, similar structural ${ }^{71}$ and functional ${ }^{20,21}$ findings have been reported for girls at risk for MDD. The limitation of these results to females as well as the much higher prevalence of MDD and PTSD in female adolescents ${ }^{72}$ is in line with the partial gender bias in our results. Not only in $\mathrm{MDD}^{73}$ but also in healthy samples, ${ }^{74}$ reduced outcomerelated ventral striatal activation has been observed as a correlate of anhedonia. Thus, adolescents with relatively 'cold' mothers might be more vulnerable to depressive symptoms.
Along similar lines, recent research suggests that related neural processes subserve depression and insecure attachment. ${ }^{75}$ However, such processes are likely to be also influenced by the child's genetic disposition. As Caspi and Moffit ${ }^{76}$ pointed out, environmental aspects (such as a lack of maternal support) can merely constitute risk factors for the emergence of psychiatric diseases because exposure to them does not always generate disorder. Therefore, the results of this study may be viewed as a facet in a more complex gene by environment interaction that could underlie the development of mental illness. Although it is conceivable that heritability has a role in our findings, mothering and its neurobiological basis have been suggested to be transmitted across generations mainly through behavior rather than through genetic processes. ${ }^{2,77}$ Hence, our findings unlikely represent a pure genetic effect.

Our study encompasses several weaknesses. First of all, affiliation scores were derived from a questionnaire originally assessing interpersonal problems, and interpersonal affiliation was not assessed as a beneficial attitude per se but as a tendency to be rather too caring and friendly than being too 'cold' and hostile. However, the affiliation score was not based on absolute values but on difference scores that reflect individual styles instead of problem severity. Moreover, previous research showing that the affiliation dimension is positively related to secure attachment as well as our findings of a positive correlation with hippocampal gray matter demonstrate the validity of our approach. Second, we did not assess attachment style in the mothers or the children. Therefore, we cannot draw definite conclusions as to how our results translate to the attachment concept. Although in the light of previous research and theoretical accounts ${ }^{33,35-37}$ it seems likely that there is a substantial connection, future studies are needed to clarify the relationship between maternal affiliation and attachment with children's neural features and attachment. In addition, the present study relies on cross-sectional data, allowing only speculation about the clinical relevance of our results. However, a plan to follow up this cohort will be implemented, clarifying the predictive value of our findings.

In summary, our findings show that maternal interpersonal style relates to adolescents' structural and functional neural properties. Our study suggests a model of how maternal interpersonal features may be linked to the development of a certain neural reward processing pattern, with possible implications for the vulnerability for psychiatric illness.

\section{Conflict of interest}

The authors declare no conflict of interest.

Acknowledgements. This work was supported by the IMAGEN project, which receives research funding from the European Community's Sixth Framework Program (Grant number LSHM-CT-2007-0372860; the coordinated project Alzheimer's Disease, Alcoholism, Memory, (Grant number ADAMS 242257); the United Kingdom National Institute for Health Research Biomedical Research Center Mental Health, the Medical Research Council Addiction Research Cluster 'Genomic Biomarkers'; the Medical Research Council program grant 'Developmental pathways into adolescent substance abuse' (Grant number 93558); and the German Ministry of Education and Research (BMBF Grant number 01EV0711). 
This manuscript reflects only our views and the Community is not liable for any use that may be made of the information contained therein. We further acknowledge the work that has been done at Neurospin, Commissariat a l'Energie Atomique, Paris, France, for the IMAGEN project. We thank A Barbot, Y Schwartz, B Thyreau, Scito and NordicNeuroLab for transferring the data, organizing, sanity, quality check and making the data available on the IMAGEN database, including a version of the preprocessed and subject by subject statistical analysis, as well as J-B Poline for coordinating and taking responsibility for the bioinformatics and biostatistics aspects.

1. Luecken L. Childhood attachment and loss experiences affect adult cardiovascular and cortisol function. Psychsom Med 1998; 60: 765-772.

2. Champagne F, Meaney MJ. Like mother, like daughter: evidence for non-genomic transmission of parental behavior and stress responsivity. Prog Brain Res 2001; 133: 287-302.

3. McEwen BS. Stress and hippocampal plasticity. Annu Rev Neurosci 1999; 22: 105-122.

4. Gianaros PJ, Jennings JR, Sheu LK, Greer PJ, Kuller LH, Matthews KA. Prospective reports of chronic life stress predict decreased grey matter volume in the hippocampus. Neuroimage 2007; 35: 795-803.

5. Pruessner JC, Dedovic K, Pruessner M, Lord C, Buss C, Collins L et al. Stress regulation in the central nervous system: evidence from structural and functional neuroimaging studies in human populations - 2008 Curt Richter Award Winner. Psychoneuroendocrinology 2010; 35: $179-191$

6. Champagne DL, Bagot RC, van Hasselt F, Ramakers G, Meaney MJ, de Kloet ER et al. Maternal care and hippocampal plasticity: evidence for experience-dependent structura plasticity, altered synaptic functioning, and differential responsiveness to glucocorticoids and stress. J Neurosci 2008; 28: 6037-6045.

7. Luby JL, Barch DM, Belden A, Gaffrey MS, Tillman R, Babb C et al. Maternal support in early childhood predicts larger hippocampal volumes at school age. Proc Natl Acad Sci USA 2012; 109: 2854-2859.

8. Quirin M, Gillath O, Pruessner JC, Eggert LD. Adult attachment insecurity and hippocampal cell density. Soc Cogn Affect Neurosci 2010; 5: 39-47.

9. Pechtel P, Pizzagalli DA. Effects of early life stress on cognitive and affective function: an integrated review of human literature. Psychopharmacology 2010; 214: 55-70.

10. Noriuchi M, Kikuchi $Y$, Senoo A. The functional neuroanatomy of maternal love: mother's response to infant's attachment behaviors. Biol Psychiatry 2008; 63: 415-423.

11. Minagawa-Kawai Y, Matsuoka S, Dan I, Naoi N, Nakamura K, Kojima S. Prefrontal activation associated with social attachment: facial-emotion recognition in mothers and infants. Cereb Cortex 2009; 19: 284-292.

12. Strathearn L, Fonagy P, Amico J, Montague PR. Adult attachment predicts maternal brain and oxytocin response to infant cues. Neuropsychopharmacology 2009; 34: 2655-2666

13. Lebreton M, Barnes A, Miettunen J, Peltonen L, Ridler K, Veijola J et al. The brain structural disposition to social interaction. Eur J Neurosci 2009; 29: 2247-2252.

14. Drevets WC. Orbitofrontal cortex function and structure in depression. Ann NY Acad Sci 2007; 1121: 499-527.

15. Koolschiin PCMP, van Haren NEM, Lensvelt-Mulders GJLM, Hulshoff Pol HE, Kahn RS Brain volume abnormalities in major depressive disorder: a meta-analysis of magnetic resonance imaging studies. Hum Brain Mapp 2009; 30: 3719-3735.

16. Parker G, Hadzi-Pavlovic D, Greenwald S, Weissman M. Low parental care as a risk facto to lifetime depression in a community sample. J Affect Disord 1995; 33: 173-180.

17. Patton GC, Coffey C, Posterino M, Carlin JB, Wolfe R. Parental 'affectionless control' in adolescent depressive disorder. Soc Psychiatry Psychiatr Epidemiol 2001; 36: 475-480.

18. Pizzagalli DA, Holmes AJ, Dillon DG, Goetz EL, Birk JL, Bogdan R et al. Reduced caudate and nucleus accumbens response to rewards in unmedicated individuals with major depressive disorder. Am J Psychiatry 2009; 166: 702-710.

19. Stoy M, Schlagenhauf F, Sterzer P, Bermpohl F, Hägele C, Suchotzki K et al. Hyporeactivity of ventral striatum towards incentive stimuli in unmedicated depressed patients normalizes after treatment with escitalopram. J Psychopharmacol 2012; 26: 677-688.

20. Gotlib IH, Hamilton JP, Cooney RE, Singh MK, Henry ML, Joormann J. Neural processing of reward and loss in girls at risk for major depression. Arch Gen Psychiatry 2010; 67: 380-387.

21. Monk CS, Klein RG, Telzer EH, Schroth EA, Mannuzza S, Moulton JL 3rd et al. Amygdala and nucleus accumbens activation to emotional facial expressions in children and adolescents at risk for major depression. Am J Psychiatry 2008; 165: 90-98.

22. Forbes EE, Ryan ND, Phillips ML, Manuck SB, Worthman CM, Moyles DL et al. Healthy adolescents' neural response to reward: associations with puberty, positive affect, and depressive symptoms. J Am Acad Child Adolesc Psychiatry 2010; 49: 162-172.

23. Morgan JK, Olino TM, McMakin DL, Ryan ND, Forbes EE. Neural response to reward as a predictor of increases in depressive symptoms in adolescence. Neurobiol Dis (in press).

24. De Bellis MD, Keshavan MS, Shifflett H, lyengar S, Beers SR, Hall J et al. Brain structures in pediatric maltreatment-related posttraumatic stress disorder: a sociodemographically matched study. Biol Psychiatry 2002; 52: 1066-1078.

25. Elman I, Lowen S, Frederick BB, Chi W, Becerra L, Pitman RK. Functional neuroimaging of reward circuitry responsivity to monetary gains and losses in posttraumatic stress disorder. Biol Psychiatry 2009; 66: 1083-1090.
26. Sullivan HS. The Interpersonal Theory of Psychiatry. Williamson Alanson White Psychiatric Foundation: New York, NY, USA, 1953

27. Leary TF. Interpersonal Diagnosis of Personality; a Functional Theory and Methodology for Personality Evaluation. Ronald Press: Oxford, UK, 1957.

28. Horowitz LM, Alden LE, Wiggins JS, Pincus AL. Inventory of Interpersonal Problems Manual. Psychological Cooperation: San Antonio, TX, USA, 2000.

29. Pincus AL, Gurtman MB, Ruiz MA. Structural analysis of social behavior (SASB): circumplex analyses and structural relations with the interpersonal circle and the five-factor model of personality. J Pers Soc Psychol 1998; 74: 1629-1645.

30. Horowitz LM, Rosenberg SE, Bartholomew K. Interpersonal problems, attachment styles, and outcome in brief dynamic psychotherapy. J Consult Clin Psychol 1993; 61: 549-560.

31. Pincus AL, Dickinson KA, Schut AJ, Castonguay LG, Bedics J. Integrating interpersonal assessment and adult attachment using SASB. Eur J Psychol Assess 1999; 15: 206-220.

32. Gallo LC, Smith TW, Ruiz JM. An interpersonal analysis of adult attachment style: circumplex descriptions, recoiled developmental experiences, self-representations, and interpersonal functioning in adulthood. J Pers 2003; 71: 141-181.

33. Neumann E, Tress W. Close relationships in childhood and adulthood from the viewpoint of structural analysis of social behavior (SASB) and attachment theory. Psychother Psychosom Med Psychol 2007; 57: 145-153.

34. Depue RA, Morrone-Strupinsky JV. A neurobehavioral model of affiliative bonding: implications for conceptualizing a human trait of affiliation. Behav Brain Res 2005; 28 : 313-395.

35. Fonagy $\mathrm{P}$, Steele $\mathrm{H}$, Steele $\mathrm{M}$. Maternal representations of attachment during pregnancy predict the organization of infant-mother attachment at one year of age. Child Dev 1991; 62: 891-905.

36. Steele $\mathrm{H}$, Steele M, Fonagy P. Associations among attachment classifications of mothers, fathers, and their infants. Child Dev 1996; 67: 541-555.

37. Huth-Bocks AC, Levendosky AA, Bogat GA, von Eye A. The impact of maternal characteristics and contextual variables on infant-mother attachment. Child Dev 2004; 75 : 480-496.

38. Buss C, Lord C, Wadiwalla M, Hellhammer DH, Lupien SJ, Meaney MJ et al. Maternal care modulates the relationship between prenatal risk and hippocampal volume in women but not in men. J Neurosci 2007; 27: 2592-2595.

39. Schumann G, Loth E, Banaschewski T, Barbot A, Barker G, Büchel C et al. The IMAGEN study: reinforcement-related behaviour in normal brain function and psychopathology. Mol Psychiatry 2010; 15: 1128-1139.

40. Goodman R, Ford T, Richards H, Gatward R, Meltzer H. The Development and Well-Being Assessment: description and initial validation of an integrated assessment of child and adolescent psychopathology. J Child Psychol Psychiatry 2000; 41: 645-655.

41. Petersen AC. Adolescent development. Annu Rev Psychol 1988; 39: 583-607.

42. Wechsler D. Wechsler Intelligence Scale for Children ${ }^{\mathbb{R}}$ - Fourth Edition (WISC ${ }^{\mathbb{R}}$-IV). Pearson Assessment: London, UK, 2004

43. Horowitz LM, Strauß B, Kordy H. Inventar zur Erfassung Interpersonaler Probleme IIP-D [German version of the Inventory of Interpersonal Problems IIP-D]. Second ed. Beltz Test: Göttingen: Germany, 2000.

44. Alden LE, Wiggins JS, Pincus AL. Construction of circumplex scales for the Inventory of Interpersonal Problems. J Pers Assess 1990; 55: 521-536.

45. Gurtman MB. Construct validity of interpersonal personality measures: the interpersonal circumplex as a nomological net. J Pers Soc Psychol 1992; 63: 105-118.

46. Gurtman MB. Trust, distrust, and interpersonal problems: a circumplex analysis. J Pers Soc Psychol 1992; 62: 989-1002.

47. Gurtman MB. Interpersonal problems and the psychotherapy context: the construct validity of the Inventory of Interpersonal Problems. Psychol Assess 1996; 8: 241-255.

48. Acton GS, Revelle W. Interpersonal personality measures show circumplex structure based on new psychometric criteria. J Pers Assess 2002; 79: 446-471.

49. Knutson B, Westdorp A, Kaiser E, Hommer D. FMRI visualization of brain activity during a monetary incentive delay task. Neuroimage 2000; 12: 20-27.

50. Ashburner J, Friston KJ. Voxel-based morphometry: the methods. Neuroimage 2000; 11: 805-821.

51. Bramen JE, Hranilovich JA, Dahl RE, Forbes EE, Chen J, Toga AW et al. Puberty influences medial temporal lobe and cortical gray matter maturation differently in boys than girls matched for sexual maturity. Cereb Cortex 2011; 21: 636-646.

52. O'Doherty J, Dayan P, Schultz J, Deichmann R, Friston K, Dolan RJ. Dissociable roles of ventral and dorsal striatum in instrumental conditioning. Science 2004; 304: 452-454.

53. Schneider S, Peters J, Bromberg U, Brassen S, Miedl SF, Banaschewski T et al. Risk taking and the adolescent reward system: a potential common link to substance abuse. Am J Psychiatry 2012; 169: 39-46.

54. Yacubian J, Gläscher J, Schroeder K, Sommer T, Braus DF, Büchel C. Dissociable systems for gain- and loss-related value predictions and errors of prediction in the human brain. J Neurosci 2006; 26: 9530-9537.

55. Engert V, Buss C, Khalili-Mahani N, Wadiwalla M, Dedovic K, Pruessner JC. Investigating the association between early life parental care and stress responsivity in adulthood. Dev Neuropsychol 2010; 35: 570-581.

56. Rolls ET, Grabenhorst F. The orbitofrontal cortex and beyond: from affect to decisionmaking. Prog Neurobiol 2008; 86: 216-244.

57. Kahnt T, Heinzle J, Park SQ, Haynes J-D. The neural code of reward anticipation in human orbitofrontal cortex. Proc Nat Acad Sci USA 2010; 107: 6010-6015. 
58. Powell JL, Lewis PA, Dunbar RIM, García-Fiñana M, Roberts N. Orbital prefrontal cortex volume correlates with social cognitive competence. Neuropsychologia 2010; 48: 3554-3562.

59. Powell J, Lewis PA, Roberts N, García-Fiñana M, Dunbar RIM. Orbital prefrontal cortex volume predicts social network size: an imaging study of individual differences in humans. Proc R Soc B 2012; 279: 2157-2162.

60. Mechelli A, Price CJ, Friston KJ, Ashburner J. Voxel-based morphometry of the human brain: methods and applications. Curr Med Imaging Rev 2005; 1: 105-113.

61. Zatorre RJ, Fields RD, Johansen-Berg H. Plasticity in gray and white: neuroimaging changes in brain structure during learning. Nat Neurosci 2012; 15: 528-536.

62. Andersen SL, Teicher MH. Delayed effects of early stress on hippocampal development. Neuropsychopharmacology 2004; 29: 1988-1993.

63. Rizzi S, Bianchi P, Guidi S, Ciani E, Bartesaghi R. Neonatal isolation impairs neurogenesis in the dentate gyrus of the guinea pig. Hippocampus 2007; 17: 78-91.

64. Helmeke C, Seidel K, Poeggel G, Bredy TW, Abraham A, Braun K. Paternal deprivation during infancy results in dendrite- and time-specific changes of dendritic development and spine formation in the orbitofrontal cortex of the biparental rodent Octodon degus. Neuroscience 2009; 163: 790-798.

65. Mourlon V, Baudin A, Blanc O, Lauber A, Giros B, Naudon L et al. Maternal deprivation induces depressive-like behaviours only in female rats. Behav Brain Res 2010; 213: 278-287.

66. Der-Avakian A, Markou A. The neurobiology of anhedonia and other reward-related deficits. Trends Neurosci 2012; 35: 68-77.

67. Martin-Soelch C, Szczepanik J, Nugent A, Barhaghi K, Rallis D, Herscovitch P et al. Lateralization and gender differences in the dopaminergic response to unpredictable reward in the human ventral striatum. Eur J Neurosci 2011; 33: 1706-1715.

68. Becker J, Perry A, Westenbroek C. Sex differences in the neural mechanisms mediating addiction: a new synthesis and hypothesis. Biol Sex Differences 2012; 3: 14.

69. Otani K, Suzuki A, Shibuya N, Matsumoto Y, Kamata M. Dysfunctional parenting styles increase interpersonal sensitivity in healthy subjects. J Nerv Ment Dis 2009; 197: 938-941.
70. Hull AM. Neuroimaging findings in post-traumatic stress disorder. Systematic review. Br J Psychiatry 2002; 181: 102-110.

71. Chen MC, Hamilton JP, Gotlib IH. Decreased hippocampal volume in healthy girls at risk of depression. Arch Gen Psychiatry 2010; 67: 270-276.

72. Merikangas KR, He J-P, Burstein M, Swanson SA, Avenevoli S, Cui L et al. Lifetime prevalence of mental disorders in U.S. adolescents: results from the National Comorbidity Survey Replication-Adolescent Supplement (NCS-A). J Am Acad Child Adolesc Psychiatry 2010; 49: 980-989.

73. Keedwell PA, Andrew C, Williams SCR, Brammer MJ, Phillips ML. The neural correlates of anhedonia in major depressive disorder. Biol Psychiatry 2005; 58: 843-853.

74. Wacker J, Dillon DG, Pizzagalli DA. The role of the nucleus accumbens and rostral anterior cingulate cortex in anhedonia: Integration of resting EEG, $f M R I$, and volumetric techniques. Neuroimage 2009; 46: 327-337.

75. Galynker II, Yaseen ZS, Katz C, Zhang X, Jennings-Donovan G, Dashnaw S et al. Distinct but overlapping neural networks subserve depression and insecure attachment. Soc Cogn Affect Neurosci (in press).

76. Caspi A, Moffitt TE. Gene-environment interactions in psychiatry: joining forces with neuroscience. Nat Rev Neurosci 2006; 7: 583-590.

77. Fleming AS, Kraemer GW, Gonzales A, Lovic V, Rees S, Melo A. Mothering begets mothering: the transmission of behavior and its neurobiology across generations. Pharmacol Biochem Behav 2002; 73: 61-75.

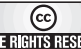

Translational Psychiatry is an open-access journal published by Nature Publishing Group. This work is licensed under the Creative Commons Attribution-NonCommercial-No Derivative Works 3.0 Unported License. To view a copy of this license, visit http://creativecommons.org/licenses/by-nc-nd/3.0/

Supplementary Information accompanies the paper on the Translational Psychiatry website (http://www.nature.com/tp) 